\title{
A geropathology approach for identifying therapeutic tar- gets to prevent pathological complications of COVID-19
}

\author{
Warren Ladiges ${ }^{\mathrm{a},{ }^{*}}$, Yuji Ikeno ${ }^{\mathrm{b}}$, Liang Wang $^{\mathrm{c}}$, Feng Liu $^{\mathrm{d}}$ \\ ${ }^{a}$ Department of Comparative Medicine, School of Medicine, University of Washington, Seattle, WA, USA. \\ ${ }^{b}$ Barshop Institute for Longevity and Aging Studies, The University of Texas Health Science Center at San Antonio, San \\ Antonio, TX, USA. \\ ${ }^{c}$ Department of Hematology, Beijing Tongren Hospital, Capital Medical University, Beijing, 100730, China. \\ ${ }^{d}$ Department of Geriatrics, National clinical key specialty, Guangzhou First People's Hospital, School of Medicine, South \\ China University of Technology; Guangzhou Medical University, Guangzhou, 510180, China.
}

\begin{abstract}
Vitally important aspects of dealing with the COVID-19 pandemic include the development of effective treatments for acute illness and prevention of infection. However, severe complications including mortality occur primarily in older adults, so a long-term approach of covering all aspects of infection and disease complications in older people is equally essential in preventing the alarming morbidity and mortality. Since severe pathological consequences of SARS-CoV-2 infection are age-related, a geropathology based platform of identifying age-related anatomic and molecular lesions aligned with processes of aging will be able to identify multiple targets for combinations of anti-aging drugs. A comprehensive geropathology-driven therapeutic approach of covering complications and vaccine effectiveness by enhancing resilience to aging will therefore play a critical role in preventing the alarming medical complications of disease not only with SARS-CoV-2 but also serve as a model for successfully controlling and preventing future world-wide pandemics.
\end{abstract}

Keywords: Resilience to aging, COVID-19 complications, SARS-CoV-2 pathology, geropathology

The immediate concern of the COVID-19 pandemic is that hundreds of thousands of people are being infected with a virus that appears to mainly attack the respiratory system so the focus has been on the immune system and the lungs. It is critical that this be addressed clinically, but we are suggesting that now is also the time to extend the scope of investigation to an organismal level and include all major organs and investigate the pathological consequences of the disease, both short term and long term, as a way to prevent infection and disease complications. The SARS-CoV-2 virus has the ability to infect people of all ages, but severe symptoms and mortality occur primarily in frail older adults who account for the majority of hospitalized patients. Why is this? Currently, in the United States approximately 80 percent of COVID-19 deaths have occurred in patients 65 years of age and older, and

\footnotetext{
* Corresponding author: Warren Ladiges

Mailing address: Department of Comparative Medicine, School of Medicine, University of Washington, Seattle, WA, USA.

E-mail: wladiges@uw.edu
}

Received: 20 May 2020 / Accepted: 26 May 2020 fatal outcomes are even more common in patients greater than 85 . Therefore, aging is a major risk factor for developing severe complications related to SARS-CoV-2 infection. An emerging platform for investigating pathological consequences of age-related disease is geropathology. Geropathology is defined as the study of the pathology of aging and considers that all anatomic and molecular lesions within an organ or tissue have a role in aging, not just those contributing to morbidity and mortality. In other words, the basic pathobiology of aging affects the functional performance of all organs in the body.

Geropathology is embraced within the field of geroscience, which aims to understand at the cellular and molecular level, interconnections between aging and disease with a focus on understanding the mechanisms by which aging contributes to disease as a major risk factor [1]. The geroscience concept posits that manipulation of aging will simultaneously delay the appearance or severity of major diseases because they share the same underlying major risk factor: aging and the multiple pathological processes involved in aging $[2,3]$. The geroscience approach assumes that all diseases that affect primarily older adults have a common and major underlying cause of declining function and resilience that is part of the aging process. 
This has been established for chronic diseases and is now a reality for acute infections such as SARS-CoV-2.

In order to answer the question "Why are older people at greater risk for severe complications of COVID-19?”, we first must address the question "How does the geropathology background influence the severity of COVID-19?" We are dealing with two issues with SARS-CoV-2 infection. The first is acute lung injury (ALI) caused by the presence of the virus, and the second is the existing presence of anatomic and molecular lesions that will influence the severity of virus/host-mediated consequences. In preclinical studies in mice, we have an ideal model to address both questions. There are scoring systems that have been adapted from human pathology and can be used for determining the severity of ALI in aging mice infected with SARS-CoV-2. Scoring systems for acute injury in other organs such as heart and brain are also being adapted for COVID-19 mouse models. This provides very useful data, but does not address the question of resilience, ie., resistance to the development of severe pathology and potential death. This is where geropathology assessment can reveal highly informative "background" details by profiling the presence and severity of anatomic and molecular lesions generally associated with increasing age. Anatomic geropathology profiles for aging mice are derived by applying a validated scoring platform to histological lesions in tissues from major organs [4]. These scores can be used for correlation analyses with acute tissue injury and other clinical pathology data. Specific cell types in lesions can also be identified using immunohistochemistry and cellspecific antibody reagents to measure quantitative staining intensity by digital imaging with programs like QuPath [5]. Molecular geropathology fingerprints are determined by DNA sequence analysis of cells identified in formalin fixed paraffin embedded tissues with specific anatomic lesions.

Once the geropathology profiles have been established and aligned with SARS-COV-2 infection, it is a matter of identifying aging processes that match up with the aligned profiles. Using the aging COVID-19 mouse model, and qualitative and quantitative platforms such as IHC and Luminex, tissues can be interrogated for processes of aging. Some important processes include inflammation, autophagy impairment, insulin/mitochondrial dysfunction and oxidative stress, epigenetic dysfunction, and vascular impairment. This approach is designed to identify aging processes that increase the risk of developing pathology with COVID-19. The processes of aging that align with COVID-19 pathology should be able to be used to define successful intervention of disease manifestation by showing evidence of cellular homeostasis in concert with immunological response and decreased geropathology. The geroscience concept posits that susceptibility to the pathologic development of COVID-19 complications with increasing age is not the result of a single aging pathway. Rather, it is a multifactorial network linked with mechanisms of aging. Therefore, it is a matter of matching the relevant aging processes with drugs that target specific molecules mechanistically involved in these processes and administering these drugs in a manner informed by the data. A number of drugs have been shown to have antiaging effects not only in preclinical studies but also in clinical investigations. Most of these are already clinically approved for other diseases such as diabetes and examples include metformin, rapamycin, acarbose, as well as others. Since the development of COVID-19 pathology is age-related, and multiple processes are involved in aging, there will not be one single drug that will prevent COVID-19 complications in the elderly. Rather, it will require combining anti-aging drugs that fit parts of the geropathology profile into combinations that target all the parts. Drug combinations that result in improvements in multiple aspects of healthy aging significantly delay the appearance of pathology and frailty [6]. In other words, there is increased resilience to aging that is more characteristic of a younger biological age compared to an older chronological age.

The real question then is "Is a more youthful biological state beneficial for alleviating pathology and fatal outcomes of COVID-19 infection in older people?" The ultimate expectation is that processes of aging gravitate towards a functional homeostasis representative of a young age and align more with the expected youthful biological profile. Observations from data generated on specific processes of aging are critical to show that a geropathology approach for alleviating severe COVID-19-related pathology is possible, and increases the validity of properly matched combinations of anti-aging drugs for enhancing and protecting elderly people from the devastating effects of SARS-CoV-2 infection. These predictions are not unreasonable or risky expectations, because drug combinations are designed to target processes of aging based on mechanistic targets of each individual drug.

There are several challenges with a geropathology driven treatment/prevention approach. The first is not knowing exactly when a drug combination should be administered to older people. Whether these types of drugs would be complimentary to other treatments at the time of illness is not known but preclinical studies could easily address this. Whether and how to treat with a drug combination before clinical illness or exposure to the virus is a very relevant question. Preclinical studies in aging mice pretreated with drug combinations for three months show delayed aging (Ladiges, et al., unpublished observations). Three mouse months is equivalent to 7 to 8 human years, which is a considerable duration for older people, but shorter prophylactic treatment times will most likely be effective. Preclinical and clinical studies could easily address this. Another related question is how to identify older people at risk for complications of COVID-19. Since severe COVID-19 pathology is an age-related condition, we can pose the question in a different way by asking who is resilient to physical aging thereby identifying people that, by definition, would be more resistant to COVID-19 complications. Stress tests are already being developed for resilience to aging, so there are potential clinical applica- 
tions for this approach. Another related challenge is how will a combination of anti-aging drugs affect older people already taking one or more drugs for specific medical conditions. In fact, many older people are polypharmacy patients, ie., they are taking five or more drugs. Aside from the fact that the anti-aging drug combination may alleviate the need for some, or all medically indicated drugs, there are ethical and clinical issues that would need to be addressed. A third challenge is determining if an antiaging drug combination would enhance the effectiveness of a SARS-CoV-2 vaccine. Although a vaccine has not yet been developed for widespread clinical use, this is a very relevant question, since it is known that immune responsiveness to vaccines generally decreases with increasing age, and some anti-aging drugs can enhance immune responses.

In summary, effective treatments for acute SARS-CoV-2 illness and prevention of infection are vitally necessary aspects of dealing with this pandemic. However, a long term geropathology approach of covering all aspects of infection and disease complications in older people is equally essential in preventing the alarming morbidity and mortality of not only the COVID-19 pandemic but also for future world-wide pandemics. Therapeutic targeting of aging processes has the potential of enhancing resilience to aging and subsequently age-related diseases including infectious disease agents thereby improving and extending healthy living with increasing age.

\section{Declarations}

Conflict of Interest: The authors declare that they have no conflict of interest.

Acknowledgements: No funding was received for the authoring of this commentary.

\section{References}

1. Sierra F, Kohanski R. Geroscience and the trans-NIH geroscience interest group, GSIG. 2017.

2. Kennedy B K, Berger S L, Brunet A, et al. Geroscience: linking aging to chronic disease. Cell, 2014, 159(4): 709713.

3. Wahl D, Anderson R M, Le Couteur D G. Antiaging Therapies, Cognitive Impairment, and Dementia. The Journals of Gerontology: Series A, 2019.

4. Snyder J M, Snider T A, Ciol M A, et al. Validation of a geropathology grading system for aging mouse studies. GeroScience, 2019, 41(4): 455-465.

5. Bankhead P, Loughrey M B, Fernández J A, et al. QuPath: Open source software for digital pathology image analysis. Scientific reports, 2017, 7(1): 1-7.

6. Ladiges $\mathrm{W}$, Liggitt $\mathrm{D}$. Testing drug combinations to slow aging. Pathobiology of Aging \& Age-related Diseases, 2017.

Cite this article as: Ladiges W, Ikeno Y, Wang L, et al. A geropathology approach for identifying therapeutic targets to prevent pathological complications of COVID-19[J]. Aging Pathobiology and Therapeutics, 2020, 2(2): 106-108. 